\title{
ON RANDOM ORTHOGONAL POLYNOMIALS
}

\author{
K. FARAHMAND \\ University of Ulster at Jordanstown \\ Department of Mathematics \\ Co. Antrim, BT37 0QB, United Kingdom \\ E-mail: K.Farahmand@ulst.ac.uk
}

(Received March, 1999; Revised September, 2000)

Let $T_{0}^{*}(x), T_{1}^{*}(x), \ldots, T_{n}^{*}(x)$ be a sequence of normalized Legendre polynomials orthogonal with respect to the interval $(-1,1)$. The asymptotic estimate of the expected number of real zeros of the random polynomial $g_{0} T_{0}^{*}(x)+g_{1} T_{1}^{*}(x)+\ldots+g_{n} T n^{*}(x)$ where $g_{j}, j=0,1, \ldots, n$ are independent identically and normally distributed random variables is known. In this paper, we first present the asymptotic value for the above expected number when coefficients are dependent random variables. Further, for the case of independent coefficients, we define the expected number of zero up-crossings with slope greater than $u$ or zero down-crossings with slope less than $-u$. Promoted by the graphical interpretation, we define these crossings as $u$-sharp. For the above polynomial, we provide the expected number of such crossings.

Key words: Sharp Crossings, Number of Real Roots, Kac-Rice Formula, Normal Density, Legendre Polynomial.

AMS subject classifications: $60 \mathrm{H} 99,42 \mathrm{BXX}$.

\section{Introduction}

Let $(\Omega, A, \operatorname{Pr})$ be a fixed probability space and $\left\{g_{j}(\omega)\right\}_{j=0}^{n}, \omega \in \Omega$, be a sequence of normally distributed random variables. Let $T_{j}(x)$ be a Legendre polynomial and $T_{j}^{*}(x)=\sqrt{(j+1 / 2)} T_{j}(x)$ be a normalized Legendre polynomial orthogonal with respect to the weight function unity. We denote $N_{n}(a, b)$ by the number of real zeros of $P_{n}(x)$ in the interval $(a, b)$ where

$$
P_{n}(x) \equiv P_{n}(x, \omega)=\sum_{j=0}^{n} g_{j}(\omega) T_{j}^{*}(x) .
$$

For the case of independent coefficients, Das [2] shows that for $n$ sufficiently large, $E N_{n}(-1,1)$, the expected number of real zeros of $P_{n}(x)$ is asymptotic to $n / \sqrt{3}$. Wilkins [13] is an interesting work which involves much delicate analysis and reduces Das' error term significantly. In more recent work [4] (see also [5]), we consider the 
case of non-identically distributed $g_{j} \mathrm{~s}$. However, all the above results were obtained by insisting on the coefficients being independent.

Motivated by interesting results obtained in [8,9] and [10] for the dependent coefficients where $T_{j}^{*}(x)$ in $(1.1)$ is defined as $x^{j}, j=0,1, \ldots, n$ as well as in order to gain a better understanding of the mathematical behavior of $P_{n}(x)$, we consider the case when the coefficients $g_{j}$ are dependent with moment matrix with $\rho_{i i}=\sigma^{2}$ and $\rho_{i j}=\rho, 0<\rho<1, i \neq j$. Comparing our results for the Legendre polynomials with the algebraic one, in the cases of independent versus dependent, significant differences in the behavior are revealed. It is shown that $E N_{n}(-\infty, \infty)$ for the algebraic case with dependent coefficients is half that of the independent case. However, we show that for our case of Legendre polynomials, the expected number of zeros is invariant for both dependent and independent cases.

In another direction, we define a real zero of $P_{n}(x, \omega)$ as $u$-sharp when it up-crosses the $x$-axis with slope greater than $u$ or down-crosses it with slope smaller than $-u$. We denote the number of $u$-sharp crossings of $P_{n}(x, \omega)$ in the interval $(a, b)$ by $S_{u}(a, b)$. Our above method allows us to show that in the case of independent coefficients, most of the crossings of random Legendre polynomials are $u$-sharp. That is, unlike algebraic cases, $E S_{u}(-1,1)$ is independent of $u$. We prove the following theorems.

Theorem 1: If the coefficients of $P_{n}(x)$ in (1.1) are dependent with the above covariance matrix and mean $\mu$ then, for all sufficiently large $n$, the expected number of real zeros of $P_{n}(x)$ is

$$
E N_{n}(-1,1) \sim \frac{n}{\sqrt{3}}
$$

Theorem 2: If the coefficients of $P_{n}(x)$ in (1.1) are independent with mean $\mu$, then for all $u$ such that $u / n^{3} \rightarrow 0$ as $n \rightarrow \infty$, the expected number of u-sharp crossings $i s$

$$
E S_{u}(-1,1) \sim \frac{n}{\sqrt{3}}
$$

\section{Analysis}

Let

$$
\begin{array}{cl}
A^{2}=\operatorname{var}\left\{P_{n}(x)\right\}, & B^{2}=\operatorname{var}\left\{P_{n}^{\prime}(x)\right\}, \\
C=\operatorname{cov}\left\{P_{n}(x), P_{n}^{\prime}(x)\right\} & \theta=\frac{\operatorname{cov}\left\{P_{n}(x), P_{n}^{\prime}(x)\right\}}{A B} \\
\lambda_{1}=E\left\{P_{n}(x)\right\} & \lambda_{2}=E\left\{P_{n}^{\prime}(x)\right\}
\end{array}
$$

and

$$
\eta_{u}=\frac{\lambda_{2}-B \theta \lambda_{1} / A+u}{B \sqrt{1-\theta^{2}}}
$$


Then from Cramér and Leadbetter [1, p. 125], the expected number of real zeros of $P_{n}(x)$ in $(a, b)$ can be obtained as

$$
E N_{n}(a, b)=\int_{a}^{b} \frac{B \sqrt{1-\theta^{2}}}{A} \phi\left(\frac{\lambda_{1}}{A}\right)\left[2 \phi\left(\eta_{0}\right)+\eta_{0}\left\{2 \Phi\left(\eta_{0}\right)-1\right\}\right] d x
$$

where $\Phi(t)$ and $\phi(t)$ are the distribution and density functions of a normal standard random variable, respectively. Denote $\Delta^{2}=A^{2} B^{2}-C^{2}$ and $\operatorname{erf}(x)=\int_{0}^{x} \exp \left(-t^{2}\right) d t$; then from (2.1) and since

$$
\Phi(x)=\frac{1}{2}+\frac{\operatorname{erf}(x / \sqrt{2})}{\sqrt{\pi}}
$$

we can write

$$
\begin{gathered}
E N_{n}(a, b)=\int_{a}^{b}\left\{\frac{\Delta}{\pi A^{2}} \exp \left(-\frac{A^{2} \lambda_{2}^{2}-2 C \lambda_{1} \lambda_{2}+B^{2} \lambda_{1}^{2}}{2 \Delta^{2}}\right)\right. \\
\left.+\frac{\sqrt{2}\left|A^{2} \lambda_{2}-C \lambda_{1}\right|}{\pi A^{3}} \exp \left(-\frac{\lambda_{1}^{2}}{2 A^{2}}\right) \operatorname{erf}\left(\frac{\left|A^{2} \lambda_{2}-C \lambda_{1}\right|}{A \Delta \sqrt{2}}\right)\right\} d x .
\end{gathered}
$$

Also with the above definition of $u$-sharp crossings from [5, p. 18], we have

$$
E S_{u}(a, b)=\int_{a}^{b} \frac{B \sqrt{1-\theta^{2}}}{A} \phi\left(\frac{\lambda_{1}}{A}\right)\left[\phi\left(\eta_{u}\right)+\phi\left(\eta_{-u}\right)+\eta_{0}\left\{\Phi\left(\eta_{u}\right)+\Phi\left(\eta_{-u}\right)-1\right\}\right] d x
$$

Now by using (2.2) and with a little algebra, we obtain the following formula for the expected number of $u$-sharp crossings:

$$
\begin{aligned}
& E S_{u}(a)=\int_{a}^{b}\left[\frac{\Delta}{2 \pi A^{2}}\left\{\exp \left(-\frac{\lambda_{1}^{2}}{2 A^{2}}-\frac{\gamma_{u}}{2}\right)+\exp \left(-\frac{\lambda_{1}^{2}}{2 A^{2}}-\frac{\gamma-u}{2}\right)\right\}\right. \\
& \left.+\frac{\left|A^{2} \lambda_{2}-C \lambda_{1}\right|}{\pi A^{2} \sqrt{2}} \exp \left(-\frac{\lambda_{1}^{2}}{2 A^{2}}\right)\left\{\operatorname{erf}\left(\frac{\left|\gamma_{u}\right|}{\sqrt{2}}\right)+\operatorname{erf}\left(\frac{\left|\gamma_{-u}\right|}{\sqrt{2}}\right)\right\}\right] d x
\end{aligned}
$$

where $\gamma_{u} \equiv \gamma_{u}(x)=\left(A \lambda_{2}-C \lambda_{1} / A+A u\right) / \Delta$. In the following we evaluate those elements that appear in formula (2.3) and (2.4). To this end, with the assumptions of the theorems, we have

$$
\begin{aligned}
& A^{2}=\left(\sigma^{2}-\rho\right) \sum_{j=0}^{n} T_{j}^{* 2}(x)+\rho\left\{\sum_{j=0}^{n} T_{j}^{*}(x)\right\}^{2} \\
& B^{2}=\left(\sigma^{2}-\rho\right) \sum_{j=0}^{n} T_{j}^{*^{\prime} 2}(x)+\rho\left\{\sum_{j=0}^{n} T_{j}^{*^{\prime}}(x)\right\}^{2}
\end{aligned}
$$




$$
\begin{gathered}
C=\left(\sigma^{2}-\rho\right) \sum_{j=0}^{n} T_{j}^{*}(x) T_{j}^{*^{\prime}}(x)+\rho \sum_{j=0}^{n} T_{j}^{*}(x) \sum_{j=0}^{n} T_{j}^{*^{\prime}}(x) \\
\lambda_{1}=\mu \sum_{j=0}^{n} T_{j}^{*}(x)
\end{gathered}
$$

and

$$
\lambda_{2}=\mu \sum_{j=0}^{n} T_{j}^{*^{\prime}}(x)
$$

In order to estimate the terms that appear in (2.5)-(2.9), we recall the following properties valid for Legendre polynomials, see for example [6, p. 1024]

$$
\begin{aligned}
\sum_{j=0}^{n}\left\{T_{j}^{*}(x)\right\}^{2} & =\frac{n+1}{2} \sqrt{\frac{2 n+3}{2 n+1}}\left\{T_{n+1}^{\prime}(x) T_{n}(x)-T_{n+1}(x) T_{n}^{\prime}(x)\right\} \\
\sum_{j=0}^{n}\left\{T_{j}^{*^{\prime}}(x)\right\}^{2} & =\frac{n+1}{12} \sqrt{\frac{2 n+3}{2 n+1}}\left\{T_{n+1}^{\prime \prime \prime}(x) T_{n}(x)-T_{n+1}(x) T_{n}^{\prime \prime \prime}(x)\right. \\
& \left.+T_{n+1}^{\prime \prime}(x) T_{n}^{\prime}(x)-T_{n+1}^{\prime}(x) T_{n}^{\prime \prime}(x)\right\}
\end{aligned}
$$

and

$$
\sum_{j=0}^{n} T_{j}^{*}(x) T_{j}^{*^{\prime}}(x)=\frac{n+1}{4} \sqrt{\frac{2 n+3}{2 n+1}}\left\{T_{n+1}^{\prime \prime}(x) T_{n}(x)-T_{n+1}(x) T_{n}^{\prime \prime}(x)\right\}
$$

As far as $\lambda_{1}$ and $\lambda_{2}$ are concerned, we will see that only their upper limits are needed and we will give these limits later. Now in order to evaluate the right-hand side of (2.10)-(2.12) we note that for Legendre polynomials we have the following well known recurrence formulae

$$
T_{n}(x)=\frac{2 n+3}{n+1} x T_{n+1}(x)-\frac{n+2}{n+1} T_{n+2}(x)
$$

and

$$
\begin{gathered}
T_{n}^{\prime}(x)=\frac{n}{1-x^{2}}\left\{T_{n-1}(x)-x T_{n}(x)\right\} \\
=\frac{n+1}{1+x^{2}}\left\{x T_{n}(x)-T_{n+1}(x)\right\}
\end{gathered}
$$

Use has been made of (2.13), written for $T_{n-1}(x)$, in order to obtain the last equation of (2.14). Now it is easily seen that, by using (2.13) and (2.14), the righthand side of $(2.10)$ can be written as

$$
\begin{gathered}
T_{n+1}^{\prime}(x) T_{n}(x)-T_{n+1}(x) T_{n}^{\prime}(x) \\
=\frac{n+1}{1-x^{2}}\left\{T_{n+1}^{2}(x)+T_{n}^{2}(x)-2 x T_{n}(x) T_{n+1}(x)\right\}
\end{gathered}
$$


Now we proceed to estimate the right-hand side of (2.15). To this end, we assume $-1+\epsilon<x<1+\epsilon$ where $\epsilon<1$ is any positive constant, arbitrary at this stage to be chosen later. From the Laplace formula [11] by setting $p=1$ for $x=\cos \gamma$ we obtain

$$
\begin{gathered}
T_{n}(\cos \gamma)=\sqrt{\frac{2}{n \pi \sin \gamma} \sum_{\nu=0}^{p-1} \frac{k !\{\Gamma(\nu+1 / 2)\}^{2} \cos \{(n+\nu+1 / 2) \gamma-(\nu+1 / 2) \pi / 2\}}{\pi \nu !(2 \sin \gamma)^{\nu} \Gamma(n+\nu+3 / 2)}} \\
\quad+o(n \sin \gamma)^{-p-1 / 2} \\
=\sqrt{\frac{2}{n \pi \sin \gamma}} \cos \left\{\left(n+\frac{1}{2}\right) \gamma-\frac{\pi}{4}\right\}+O\left\{(n \sin \gamma)^{-3 / 2}\right\} .
\end{gathered}
$$

Therefore, we can obtain the right-hand side of (2.15) as

$$
T_{n+1}^{2}(x)+T_{n}^{2}(x)-2 x T_{n}(x) T_{n+1}(x)=\frac{2 \sqrt{1-x^{2}}}{n \pi}+O\left(\frac{1}{n\left(1-x^{2}\right)}\right) .
$$

Hence from (2.10), (2.15) and (2.16), we get

$$
\sum_{j=0}^{n}\left\{T_{j}^{*}(x)\right\}^{2}=\frac{(n+1)^{2} \sqrt{2 n+3}}{n \pi \sqrt{(2 n+1)\left(1-x^{2}\right)}}+O\left(\frac{1}{n\left(1-x^{2}\right)^{2}}\right) .
$$

In order to obtain the second term of $A^{2}$ in $(2.5)$ as well as estimating $\lambda_{1}$, we use the identity

$$
(1-x) \sum_{j=0}^{n}(2 j+1) T_{j}(x)=(n+1)\left\{T_{n}(x)-T_{n+1}(x)\right\} .
$$

Then since

we can write

$$
T_{n}(x)<\frac{4}{\sqrt{n \sqrt{1-x^{2}}}}
$$

$$
\begin{aligned}
\sum_{j=0}^{n} T_{j}^{*}(x)= & \left\{\sum_{j=0}^{p}\left(j+\frac{1}{2}\right) T_{j}(x)+\sum_{j=p+1}^{n} \frac{j+1}{(p+1 / 2)^{2}} T_{j}(x)\right\} \\
= & O\left\{(p+1) \frac{\left|T_{p}(x)-T_{p+1}(x)\right|}{1-x}\right. \\
+ & \left.(n+1) \frac{\left.\mid T_{n}(x)-T_{n+1}(x)\right\}}{\sqrt{p}(1-x)}\right\} \\
= & O\left\{\frac{n^{1 / 6}}{(1-x) \sqrt{1-x^{2}}}\right\}
\end{aligned}
$$

where $p$ stands for the integer part of $n^{2 / 3}$. Now $(2.20)$ can, indeed, be used as an upper limit for $\lambda_{1}$ as well as from it, (2.5) and (2.17) yield 


$$
\begin{aligned}
& A^{2}=\frac{\left(\sigma^{2}-\rho\right)(n+1)^{2}(2 n+3)^{1 / 2}}{\pi n(2 n+1)^{1 / 2}\left(1-x^{2}\right)^{1 / 2}} \\
& +O\left\{\frac{\sigma^{2}-\rho}{n\left(1-x^{2}\right)^{2}}+\frac{\rho n^{1 / 3}}{(1-x)^{2}\left(1-x^{2}\right)}\right\} .
\end{aligned}
$$

In order to evaluate $B^{2}$ and $C$, we note that any Legendre polynomial satisfies

$$
\frac{d^{2} y}{d x^{2}}-\left(\frac{2 x}{1-x^{2}}\right) \frac{d y}{d x}+\frac{n(n-1) y}{1-x^{2}}=0 \text {. }
$$

Therefore, the second derivative of $T_{n}^{\prime \prime}(x)$ satisfies

$$
\left(1-x^{2}\right) T_{n}^{\prime \prime}(x)=2 x T_{n}^{\prime}(x)-n(n+1) T_{n}(x) .
$$

This relation and its equivalent written for $n+1$ leads us to

$$
\begin{gathered}
T_{n+1}^{\prime \prime}(x) T_{n}^{\prime}(x)-T_{n+1}^{\prime}(x) T_{n}^{\prime \prime}(x) \\
=\frac{-(n+1)\left\{n T_{n+1}(x) T_{n}^{\prime}(x)-n T_{n+1}^{\prime}(x) T_{n}(x)+2 T_{n+1}(x) T_{n}^{\prime}(x)\right\}}{1-x^{2}}
\end{gathered}
$$

and

$$
\begin{gathered}
T_{n+1}^{\prime \prime}(x) T_{n}(x)-T_{n+1}(x) T_{n}^{\prime \prime}(x) \\
=\frac{\left.2 x T_{n+1}^{\prime}(x) T_{n}(x)-2 x T_{n+1}(x) T_{n}^{\prime}(x)-2(n+1) T_{n}(x) T_{n+1}(x)\right\}}{1-x^{2}}
\end{gathered}
$$

Now by using the first theorem of Steilzer [11, p. 127], $T_{n}(x) \leq 4 n^{-1 / 2}(1-$ $\left.x^{2}\right)^{-1 / 4}=o\left\{n^{-1 / 2}\left(1-x^{2}\right)^{-1 / 4}\right\}$. Therefore using (2.14), we obtain

$$
T_{n}^{\prime}(x)=o\left\{n^{1 / 2}\left(1-x^{2}\right)^{-5 / 4}\right\}
$$

Substituting this estimate in (2.22) and (2.23) yields

$$
\begin{gathered}
T_{n+1}^{\prime \prime}(x) T_{n}^{\prime}(x)-T_{n+1}^{\prime}(x) T_{n}^{\prime \prime}(x) \\
=\frac{n(n+1)\left\{T_{n+1}^{\prime}(x) T_{n}(x)-T_{n+1}(x) T_{n}^{\prime}(x)\right\}}{1-x^{2}}+O\left\{\frac{n}{\left(1-x^{2}\right)^{5 / 2}}\right\}
\end{gathered}
$$

and

$$
\begin{gathered}
T_{n+1}^{\prime \prime}(x) T_{n}(x)-T_{n+1}(x) T_{n}^{\prime \prime}(x) \\
=\frac{2 x}{1-x^{2}}\left\{T_{n+1}^{\prime}(x) T_{n}(x)-T_{n+1}(x) T_{n}^{\prime}(x)\right\}+O\left\{\frac{1}{\left(1-x^{2}\right)^{3 / 2}}\right\}
\end{gathered}
$$


Also, by differentiating both sides of (2.23) and using (2.22) we can obtain

$$
\begin{gathered}
T_{n+1}^{\prime \prime \prime}(x) T_{n}(x)-T_{n}^{\prime \prime \prime}(x) T_{n+1}(x) \\
=\frac{8 x^{2}+n(n+1)\left(1-x^{2}\right)}{\left(1-x^{2}\right)^{2}}\left\{T_{n+1}^{\prime}(x) T_{n}(x)-T_{n+1}(x) T_{n}^{\prime}(x)\right\}+O\left\{\frac{1}{\left(1-x^{2}\right)^{5 / 2}}\right\} .
\end{gathered}
$$

Hence (2.25) and (2.26) give an estimate for (2.11) which completes the evaluation of the first term of $B^{2}$ in (2.6). For the second term we use (2.18) and similar to $(2.20)$ we obtain

$$
\begin{aligned}
\sum_{j=0}^{n} T_{j}^{*^{\prime}}(x) & =O\left\{\frac{\sqrt{n}}{1-x^{2}} \sum_{j=0}^{n}(2 j+1) T_{j}(x)\right\} \\
& =O\left\{\frac{n^{3 / 2}\left|T_{n}(x)\right|}{\left(1-x^{2}\right)(1-x)}\right\} \\
& =O\left\{\frac{n}{(1-x)\left(1-x^{2}\right)^{5 / 4}}\right\} .
\end{aligned}
$$

Therefore from $(2.6),(2.11),(2.15),(2.16)$, and (2.25)-(2.27) we have

$$
\begin{aligned}
& B^{2}=\left(\sigma^{2}-\rho\right) \frac{(n+1)^{3}(2 n+3)^{1 / 2}}{3 \pi(2 n+1)^{1 / 2}\left(1-x^{2}\right)^{3 / 2}} \\
& +O\left\{\frac{\left(\sigma^{2}-\rho\right) n^{2}}{\left(1-x^{2}\right)^{5 / 2}}+\frac{\rho n^{2}}{(1-x)^{2}\left(1-x^{2}\right)^{5 / 2}}\right\} .
\end{aligned}
$$

Also (2.7), (2.12), (2.20) and (2.27) yields

$$
C=O\left\{\frac{\left(\sigma^{2}-\rho\right) n}{\left(1-x^{2}\right)^{3 / 2}}+\rho \frac{n^{7 / 6}}{(1-x)^{2}\left(1-x^{2}\right)^{7 / 4}}\right\}
$$

From (2.20) and (2.27), we easily obtain the following estimates for $\lambda_{1}$ and $\lambda_{2}$ as

and

$$
\lambda_{1}=o\left\{\mu \frac{n^{1 / 6}}{(1-x)\left(1-x^{2}\right)^{1 / 2}}\right\}
$$

$$
\lambda_{2}=o\left\{\mu \frac{n}{(1-x)\left(1-x^{2}\right)^{5 / 4}}\right\}
$$

\section{Proofs of Theorems}

The above estimates obtained are all valid for $x \in(-1+\epsilon, 1-\epsilon)$ for which we use the result obtained in (2.3) and (2.4) for Theorem 1 and 2, respectively. For the 
expected number of zeros outside this interval, we ought to use a completely different approach based on an application of Jensen's theorem. We will see that for the choice of $\epsilon$, any positive value smaller than $n^{-1 / 4}$ will serve our purpose, and we chose $\epsilon=n^{-1 / 4}$. First from $(2.3),(2.21)$ and (2.28)-(2.31) we note that

$$
\begin{aligned}
E N_{n}(-1+\epsilon, 1-\epsilon) & \sim \frac{n}{\pi \sqrt{3}} \int_{-1+\epsilon}^{1-\epsilon} \frac{d x}{\sqrt{1-x^{2}}} \\
& \sim \frac{n}{\sqrt{3}} .
\end{aligned}
$$

Also from (2.4), (2.21) and (2.28)-(2.31) by the assumptions given for Theorem 2 for $u$, we obtain

$$
\begin{aligned}
E S_{u}(-1+\epsilon, 1-\epsilon) & \sim \frac{n}{\pi \sqrt{3}} \int_{-1+\epsilon}^{1-\epsilon} \frac{\exp \left\{-u n^{-3}\left(1-x^{2}\right)^{3 / 2}\right\}}{\sqrt{1-x^{2}}} d x \\
& \sim \frac{n}{\pi \sqrt{3}} \int_{-1+\epsilon}^{1-\epsilon} \frac{d x}{\sqrt{1-x^{2}}} \sim \frac{n}{\sqrt{3}} .
\end{aligned}
$$

Now for both Theorem 1 and 2 we show that for $(-1,-1+\epsilon)$ and $(1-\epsilon, 1)$ the expected number of real zeros and $u$-sharp crossings is small. To this end, we use an application of Jensen's theorem [7, 12], first used by Dunnage, and its generalization to the dependent case in [3].

In order to avoid duplication, we note that the expected number of $u$-sharp crossings is smaller than the expected number of overall real zeros. Therefore, we only concentrate on the upper bound for the number of real zeros. The results for the number of sharp crossing then will follow. Let $N(r) \equiv N(r, \omega)$ denote the number of real zeros of $P(z, \omega)$ in $|z-1|<\epsilon$. Assuming $P(1) \neq 0$ from Jensen's theorem, we have

Now since

$$
N(\epsilon) \log 2 \leq \frac{1}{2 \pi} \int_{0}^{2 \pi} \log \left|\frac{P_{n}\left(1+2 \epsilon e^{i x}, \omega\right)}{P_{n}(1)}\right| d x .
$$

$$
P_{n}(z)=\frac{1}{\pi} \int_{0}^{\pi}\left\{z+i \sqrt{1-z^{2}} \cos \theta\right\}^{n} d \theta
$$

for all sufficiently large $n$, we obtain

$$
P_{n}\left(1+\epsilon e^{i x}\right) \leq(1+3 \epsilon)^{n}<\exp (n \epsilon)
$$

Therefore, since by Schward's inequality

$$
\sum_{j=0}^{n} \sqrt{j+1 / 2} \leq \sqrt{(n+1) \sum_{j=0}^{n}(j+2)}<n^{3 / 2}
$$

for all sufficiently large $n$, we can write 


$$
P_{n}\left(1+\epsilon e^{i x}\right)<n^{3 / 2} \exp (3 n \epsilon) \max \left|g_{j}\right|
$$

where the maximum is taken over $0 \leq j \leq n$. Now since $g_{j}, j=0,1,2, \ldots, n$ has a normal distribution

$$
\begin{gathered}
\left.\operatorname{Pr}\left(\max \left|g_{j}\right|\right)>n\right) \leq n \operatorname{Pr}\left(\left|g_{j}\right|>n\right) \\
=\frac{n \sqrt{2 \pi}}{\sigma} \int_{n}^{\infty} \exp \left\{-\frac{(t-\mu)^{2}}{2 \sigma^{2}}\right\} d t \\
\leq \sqrt{\frac{2}{\pi \sigma^{2}}} \exp \left\{-\frac{(n-\mu)^{2}}{2 \sigma^{2}}\right\} .
\end{gathered}
$$

Also since the distribution of $P(1)=\sum_{j=0}^{n}(\sqrt{j+1 / 2}) g_{j}$ is normal with mean $m=\mu \sum_{j=0}^{n} \sqrt{j+1 / 2}$ and variance $\Lambda^{2}=\sigma^{2} n(n+2) / 2$ we can say

$$
\begin{aligned}
\operatorname{Pr}(-1<P(1)<1)= & \frac{1}{\Lambda \sqrt{2 \pi}} \int_{-1}^{1} \exp \left\{-\frac{(t-m)^{2}}{2 \Lambda^{2}}\right\} d t \\
& <\sqrt{\frac{2}{\pi \Lambda^{2}}}
\end{aligned}
$$

Therefore, from (3.3)-(3.6) and except for sample functions in an $\omega$ set of measure not exceeding $2 / \Lambda+4 \exp \left\{-(n-\mu)^{2} / 2 \sigma^{2}\right\}<4 / n \sigma$ we have

$$
N(\epsilon)<\frac{(5 / 2) \log n+3 n \epsilon}{\log 2} .
$$

This gives $O(n \epsilon+\log n)$ as an upper bound for $E N(\epsilon)$, which is sufficient to give the proof of the theorems.

\section{References}

[1] Cramér, H. and Leadbetter, M.R., Stationary and Related Stochastic Processes, Wiley, New York 1967.

[2] Das, M., Real zeros of a random sum of orthogonal polynomial, Proc. Amer. Math. Soc. 27 (1971), 147-153.

[3] Farahmand, K., Level crossings of a random trigonometric polynomial with dependent coefficients, J. Austral. Math. Soc. 58 (1995), 39-46.

[4] Farahmand, K., Level crossings of a random orthogonal polynomial, Analysis 16 (1996), 245-253.

[5] Farahmand, K., Topics in Random Polynomials, Addison-Wesley Longman, London 1998.

[6] Gradshteyn, I.S. and Ryzhik, I.M., Table of Integrals, Series and Products, Academic Press, London 1980. 
[7] Rudin, W., Real and Complex Analysis, McGraw-Hill 1974.

[8] Sambandham, M., On the real roots of the random algebraic polynomial, Indian J. Pure Appl. Math. 7 (1976), 1062-1070.

[9] Sambandham, M., On the upper bound of the number of real zeros of a class of random algebraic equation, J. Indian Math. Soc. 42 (1978), 15-26.

[10] Sambandham, M., On the average number of real zeros of a class of random algebraic curves, Pacific J. Math. 81 (1979), 207-215.

[11] Sansone, G., Orthogonal Functions, Zanichelli, Bologna: English Transl. Pure and Appl. Math., Intersciences 9, 1952.

[12] Titchmarsh, E.C., The Theory of Functions, Oxford University Press 1939.

[13] Wilkins, J.E., The expected value of the number of real zeros of a random sum of Legendre polynomials, Proc. Amer. Math. Soc. 125 (1997), 1531-1536. 


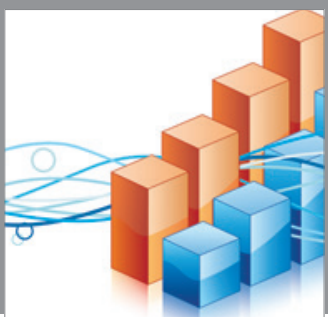

Advances in

Operations Research

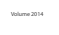

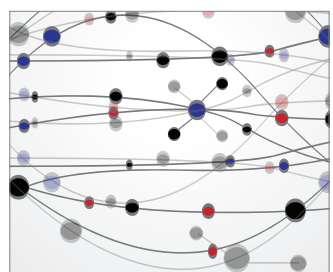

\section{The Scientific} World Journal
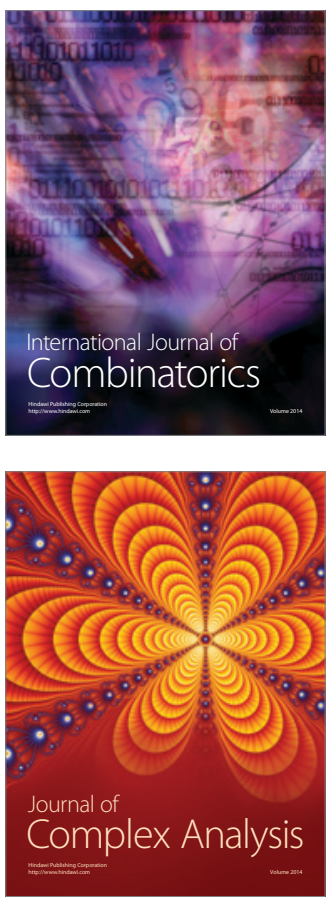

International Journal of

Mathematics and

Mathematical

Sciences
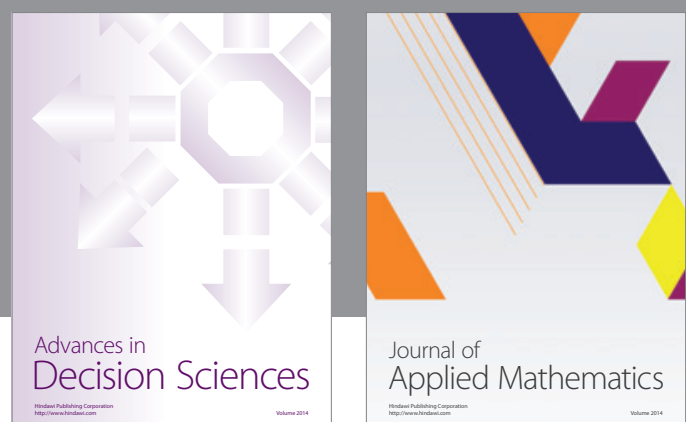

Journal of

Applied Mathematics
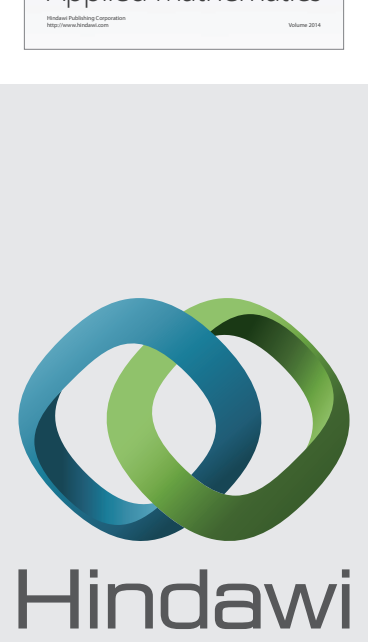

Submit your manuscripts at http://www.hindawi.com
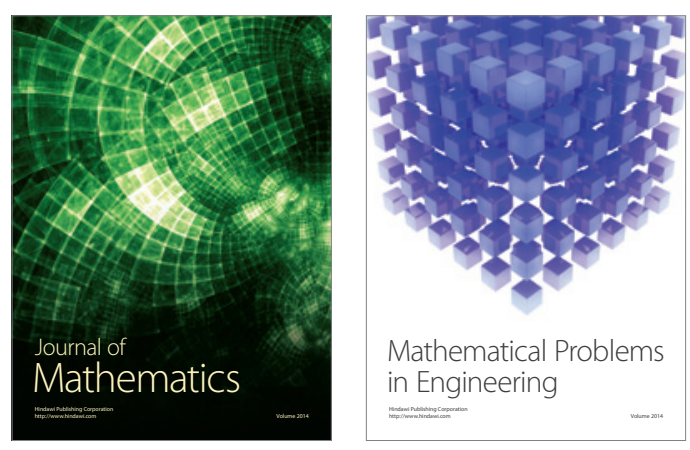

Mathematical Problems in Engineering
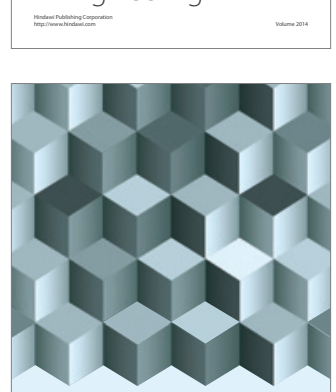

Journal of

Function Spaces
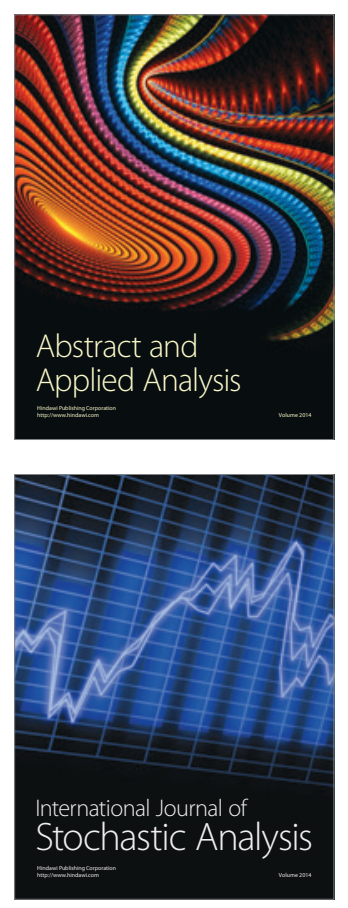

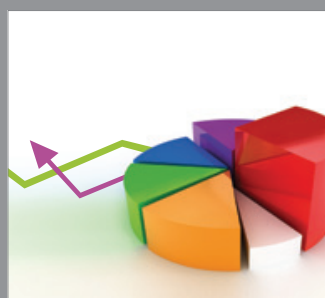

ournal of

Probability and Statistics

Promensencen
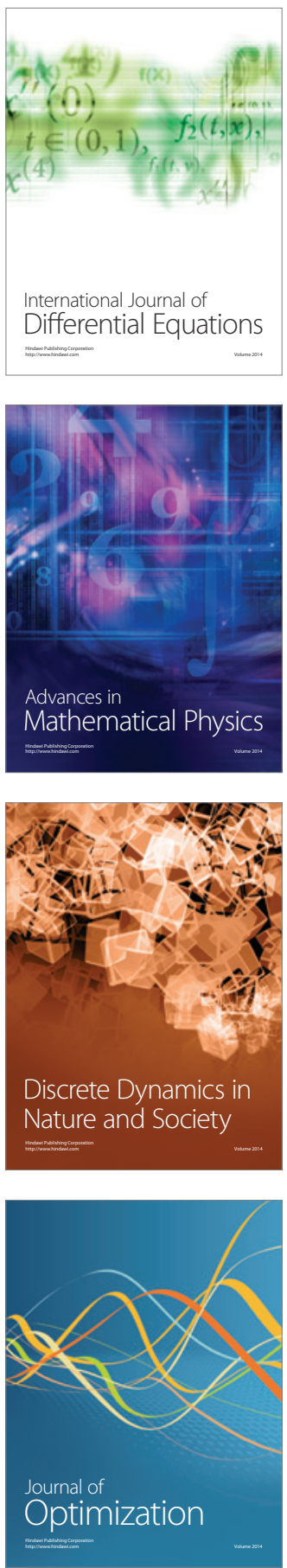